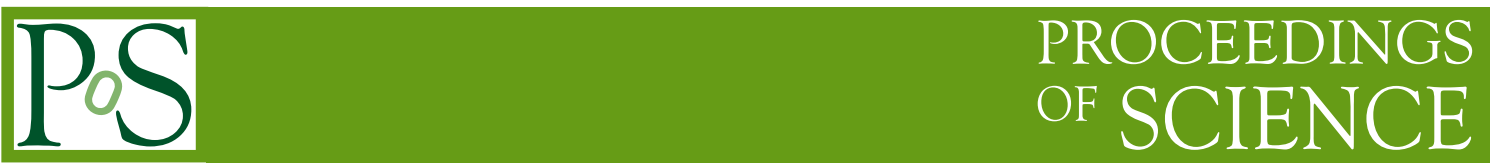

\title{
Anomaly at finite density and chiral fermions on lattice
}

\section{Rajiv V. Gavai*}

Department of Theoretical Physics, Tata Institute of Fundamental Research, Homi Bhabha Road, Mumbai 400005, India.

E-mail: gavai@tifr.res.in

\section{Sayantan Sharma ${ }^{\dagger}$}

Department of Theoretical Physics, Tata Institute of Fundamental Research, Homi Bhabha Road, Mumbai 400005, India

E-mail:ssharma@theory.tifr.res.in

\begin{abstract}
Using both perturbation theory in the Euclidean formalism as well as the non-perturbative Fujikawa's method, we verify that the chiral anomaly equation remains unaffected in continuum QCD in the presence of nonzero chemical potential, $\mu$. We extend our considerations to lattice fermions with exact chiral symmetry and discuss the consequences for the recent Bloch-Wettig proposal for the Dirac operator at finite chemical potential. We propose a new simpler method of incorporating $\mu$.
\end{abstract}

The XXVII International Symposium on Lattice Field Theory

July 26-31, 2009

Peking University, Beijing, China

\footnotetext{
${ }^{*}$ Speaker.

${ }^{\dagger}$ Supported by the Shyamaprasad Mukherjee Fellowship of CSIR, India.
} 


\section{Introduction}

Since there are an equal number of left-handed and right-handed particles in the lattice fermion propagator for each set of quantum numbers, there is no chiral anomaly on the lattice for the nave fermions. Canonical local formulations, such as the Wilson fermions, the Kogut-Susskind fermions, the twisted mass fermions or the Creutz-Boriçi fermions break the flavour singlet axial $\mathrm{U}(1)$ and some or all of the flavour non-singlet axial symmetries. The overlap fermions, on the other hand, preserve all chiral symmetries and have an index theorem as well.

Interestingly, chiral anomaly plays a crucial role in deciding the structure of the QCD phase diagram for our world with two light quarks and one moderately heavy quark. A fundamental aspect of the QCD phase diagram may be the existence of a critical point in the $T$ - $\mu_{B}$ plane, where $\mu_{B}$ denotes the baryonic chemical potential. The critical point is expected on the basis of chiral symmetries and model considerations. Since we employ lattice techniques to investigate the phase diagram, the presence on those chiral symmetries on the lattice is desired, if not required, in the entire $T-\mu_{B}$ plane. The popular staggered fermions have a remnant chiral symmetry on the lattice but a not so well-defined flavour number; two flavours are simulated using a square root of the fermion determinant for them. Clearly, use of overlap fermions with exact chiral symmetry on lattice is desirable.

Introduction of chemical potential for the overlap fermions turns out to be nontrivial. Ideally, one should construct the conserved charge, $N$, as a first step and then simply add $\mu N$ to the action. For the local fermions, this leads to $a^{-2}$ divergences in the continuum limit in thermodynamic quantities such as the energy density or the number density. One uses the freedom of adding irrelevant terms to cure them by multiplying gauge links in positive/negative time direction by $\exp (a \mu)$ and $\exp (-a \mu)$ respectively or more general functions which satisfy certain conditions [1]. Note that this modification, indeed even the simple addition of $\mu N$ does not change the chiral invariance of the lattice action in anyway. The non-local nature of the overlap fermions makes this construction difficult, possibly even non-unique [2]. It was proposed by Bloch and Wettig [3] that the same prescription of multiplying the gauge links, as above, may be used. This required them to extend the definition of the sign function employed in the overlap operator since the argument of the sign function, namely the Wilson-Dirac matrix, was no more $\gamma_{5}$-hermitian.

Banerjee, Gavai and Sharma [4] showed analytically that this prescription does not have any $a^{-2}$ divergences in the continuum limit but unfortunately the resultant overlap fermion action has no chiral invariance on the lattice for nonzero $\mu$. Of course, it is restored in the continuum limit but so it is for the simpler local Wilson fermions: in both cases the bare parameters of the action such as the quark mass or the chemical potential will get renormalized and depend on the coupling g. Alternatively, one can modify the chiral transformation, again by terms which vanish in the continuum limit, to restore the chiral invariance. This explains the results obtained by Bloch and Wettig that one even has an index theorem for the $\mu$-dependent overlap Dirac operator: the anomaly equation itself is $\mu$-dependent [3]. Our work presented in this talk was sparked by the curiosity about the fate of the anomaly relation in the continuum on the introduction of nonzero chemical potential.

Before presenting our work let us remark, however, that the change in the chiral transformation on the lattice implied by the Bloch-Wettig proposal is undesirable since it makes the generators of 
the transformation to be non-hermitian. Worse still, the symmetry group itself changes for each and every change of $\mu$, making this avenue not very useful for the original problem of investigation of the QCD phase diagram in the $T-\mu_{B}$ plane. In order to appreciate the loss, recall that the symmetry group remains the same at each $T$ for $\mu=0$. Therefore, a study of the temperature dependence of the order parameter $\langle\bar{\psi} \psi\rangle$, and any abrupt change in it at the transition temperature, can reveal the chiral symmetry restoring transition, if any, and thereby the change in the nature of the vacuum. Were the symmetry group to change as a function of $T$, it would be impossible to attribute a vanishing chiral condensate uniquely as a change in the vacuum or a phase change, thereby eliminating the role of the chiral condensate as the order parameter. Thus one can indeed use the freedom of adding terms irrelevant in the continuum limit to modify the chiral transformation on the lattice but such a change should not rob one off the usual order parameter for studying the chiral transition.

\section{Anomaly at $T=0$ and $\mu \neq 0$ in continuum}

Classically $\psi^{\prime}=\exp \left(i \alpha \gamma_{5}\right) \psi$ and $\bar{\psi}^{\prime}=\bar{\psi} \exp \left(i \alpha \gamma_{5}\right)$ is a symmetry for the QCD action for massless quarks, leading to the current conservation equation $\partial_{\mu} J_{5}^{\mu}=0$. At finite temperature and/or density, this classical symmetry remains intact. Quantum loop effects can, and do, cause corrections already at $T=\mu=0$, leading to the anomaly equation. One needs to compute $\left\langle\partial_{\mu} J_{5}^{\mu}\right\rangle$ to check this. The famous calculation of the Adler-Bell-Jackiw(ABJ) triangle diagram for the $U(1)$ case [5] demonstrated perturbatively that the axial $U(1)$ is broken by quantum effects while Fujikawa [6] provided a new insight by showing that the anomaly arises due to the change of the fermion measure under the corresponding transformation of the fermion fields in the path integral method. We have used both the methods to check the fate of the anomaly equation at finite density, i.e, on introduction of a nonzero chemical potential $\mu$ at zero temperature.

\subsection{Perturbative calculation}

The lowest order diagram is the canonincal ABJ axial vector-vector-vector(AVV) triangle diagram. It is well-known that the higher order diagrams do not contribute to the anomaly equation at zero density, neither do other diagrams like the square and pentagon diagrams. We too therefore compute only AVV triangle diagram at finite density. Our notation for the QCD Lagrangian in the Euclidean space with the finite number density term is the same as in [7]. In order to maintain consistency with the lattice literature, we have, however, chosen the Dirac gamma matrices to be Hermitian, leading to the action,

$$
\mathscr{L}=-\bar{\psi}(\not D+m) \psi-\frac{1}{2} \operatorname{Tr} F_{\alpha \beta} F_{\alpha \beta}+\mu \bar{\psi} \gamma_{4} \psi,
$$

where $\not D=\gamma_{v}\left(\partial_{v}-i g A_{v}^{a} T_{a}\right)$ with $T_{a}$ being the generators of the $\mathrm{SU}(3)$ gauge group. The $\gamma_{5}=$ $\gamma_{1} \gamma_{2} \gamma_{3} \gamma_{4}$ is also hermitian in our case. Using the canonical Euclidean space Feynman rules, the amplitude of these AVV triangle diagrams can be computed. Denoting by $\Delta^{\lambda \rho \sigma}\left(k_{1}, k_{2}\right)$ the total amplitude and contracting it with $q_{\lambda}$, the $\left\langle\partial_{\mu} j_{\mu}^{5}\right\rangle$ for $\mu \neq 0$ can be obtained from the triangle diagrams for $\mu \neq 0$ :

$$
q_{\lambda} \Delta^{\lambda \rho \sigma}=-i g^{2} \operatorname{tr}\left[T^{a} T^{b}\right] \int \frac{d^{4} p}{(2 \pi)^{4}} \operatorname{Tr}\left[\gamma^{5} \frac{1}{\not p-q-i \mu \gamma^{4}} \gamma^{\sigma} \frac{1}{\not p-\not k_{1}-i \mu \gamma^{4}} \gamma^{\rho}\right.
$$




$$
\begin{aligned}
& -\gamma^{5} \frac{1}{\not p-i \mu \gamma^{4}} \gamma^{\sigma} \frac{1}{\not p-\not \alpha_{1}-i \mu \gamma^{4}} \gamma^{\rho}+\gamma^{5} \frac{1}{\not p-q-i \mu \gamma^{4}} \gamma^{\rho} \frac{1}{\not p-\not / 2-i \mu \gamma^{4}} \gamma^{\sigma} \\
& \left.-\gamma^{5} \frac{1}{\not p-i \mu \gamma^{4}} \gamma^{\rho} \frac{1}{\not p-\not \alpha_{2}-i \mu \gamma^{4}} \gamma^{\sigma}\right] .
\end{aligned}
$$

An inspection of the eq.(2.2) reveals quadratically divergent integrals which differ from the usual case by merely having $\mu \neq 0$. The computation therefore can be done the same way by writing [8] $q_{\lambda} \Delta^{\lambda \rho \sigma}=(-i) \operatorname{tr}\left[T^{a} T^{b}\right] g^{2} \int \frac{d^{4} p}{(2 \pi)^{4}}\left[f\left(p-k_{1}, k_{2}\right)-f\left(p, k_{2}\right)+f\left(p-k_{2}, k_{1}\right)-f\left(p, k_{1}\right)\right]$ for a suitably chosen function $f$ and introducing a gauge-invariant cut-off. The nonzero $\mu$ appears in $f$ in the denominator as $\left[\left(p_{4}-i \mu\right)^{2}+\vec{p}^{2}\right]$ with linear in $\mu$ and a $\mu$-independent term in the numerator; the $\mu^{2}$ term vanishes being proportional to $\operatorname{Tr}\left[\gamma^{5} \gamma^{4} \gamma^{\sigma} \gamma^{4} \gamma^{\rho}\right] \sim \varepsilon^{4 \sigma 4 \rho}$. The final result turns [8] out to be the same anomaly relation as for $\mu=0$, since the $\mu$-dependent terms appear with $\Lambda^{-1}$, and vanish as the cut-off $\Lambda \rightarrow \infty$. The same computation is easily generalized [8] to nonzero temperatures.

\subsection{Non-perturbative calculation}

Fujikawa [6] taught us how to compute the chiral anomaly non-perturbatively using the path integral formalism. Under the chiral transformation of the fermion fields, the measure changes as

$$
\mathscr{D} \bar{\psi}^{\prime} \mathscr{D} \psi^{\prime}=\mathscr{D} \bar{\psi} \mathscr{D} \psi \operatorname{Det}\left|\frac{\partial\left(\bar{\psi}^{\prime}, \psi^{\prime}\right)}{\partial(\bar{\psi}, \psi)}\right|=\mathscr{D} \bar{\psi} \mathscr{D} \psi \exp \left(-2 i \alpha \int d^{4} x \operatorname{Tr} \gamma_{5}\right)
$$

The trace can be computed using the the eigenvectors of the operator $\not D$. It is an anti-Hermitian operator for $\mu=0$ with purely imaginary eigenvalues and the corresponding eigenvectors form a complete orthonormal basis. Using the fact $\left\{\gamma_{5}, \not D\right\}=0$, it is easy to show [8] that $\operatorname{Tr} \gamma_{5}=0$ for the space of eigenvectors with nonzero eigenvalues, leading to the usual $\operatorname{Tr} \gamma_{5}=n_{+}-n_{-}$relation.

$\not D(\mu)$ still anti-commutes with $\gamma_{5}$ but has both an anti-Hermitian and a Hermitian term. Remarkably, it turns out [8] still to be diagonalizable with right and left eigenvectors which together form a complete set using which essentially the same argument as above leads to the same $\operatorname{Tr} \gamma_{5}=n_{+}-n_{-}$for $\mu \neq 0$ as well. Note that the zero modes are still defined with respect to $\not D(\mu=0)$. This should be contrasted with the index theorem for the overlap operator proposed by Bloch-Wettig where the zero modes are $\mu$-dependent [3]. This is perhaps natural since the corresponding chiral transformation is $\mu$-dependent, as discussed above.

Curiously, a "gauge-like" symmetry, defined by a non-unitary transformation of the fermion fields, given by $\psi^{\prime}(\mathbf{x}, \tau)=\exp (\mu \tau) \psi(\mathbf{x}, \tau), \bar{\psi}^{\prime}(\mathbf{x}, \tau)=\bar{\psi}(\mathbf{x}, \tau) \exp (-\mu \tau)$, eliminates all the $\mu$ dependent terms of the QCD action. This transformation commutes with the chiral transformations, explaining thus the preservation of the same anomaly relation for $\mu \neq 0$. If one were to insist on this symmetry on the lattice, then addition of a simple $\mu N$ is easily seen to be forbidden for any local fermion action. Moreover, it can be easily implemented for any such local action, and leads naturally to all the known proposals [1] of introduction of the chemical potential on the lattice so far.

While the symmetry clearly arises in the continuum due to the local nature of the fermion action, one may choose to demand it for even non-local lattice actions. A natural proposal then for the overlap operator for nonzero $\mu$ is $D_{o v}(\mu)=\exp (-\mu \tau) D_{o v}(\mu=0) \exp (\mu \tau)$. While this 
can be shown to i) satisfy the Ginsparg-Wilson relation [9] and ii) lead divergence-free ideal gas results in the continuum limit, it clearly does not commute with the nonlocal chiral transformations unless they are made $\mu$-dependent too. Its main advantage though could still be that the usual sign function is used in this overlap operator.

\section{Two simple ideas for the lattice QCD at finite density}
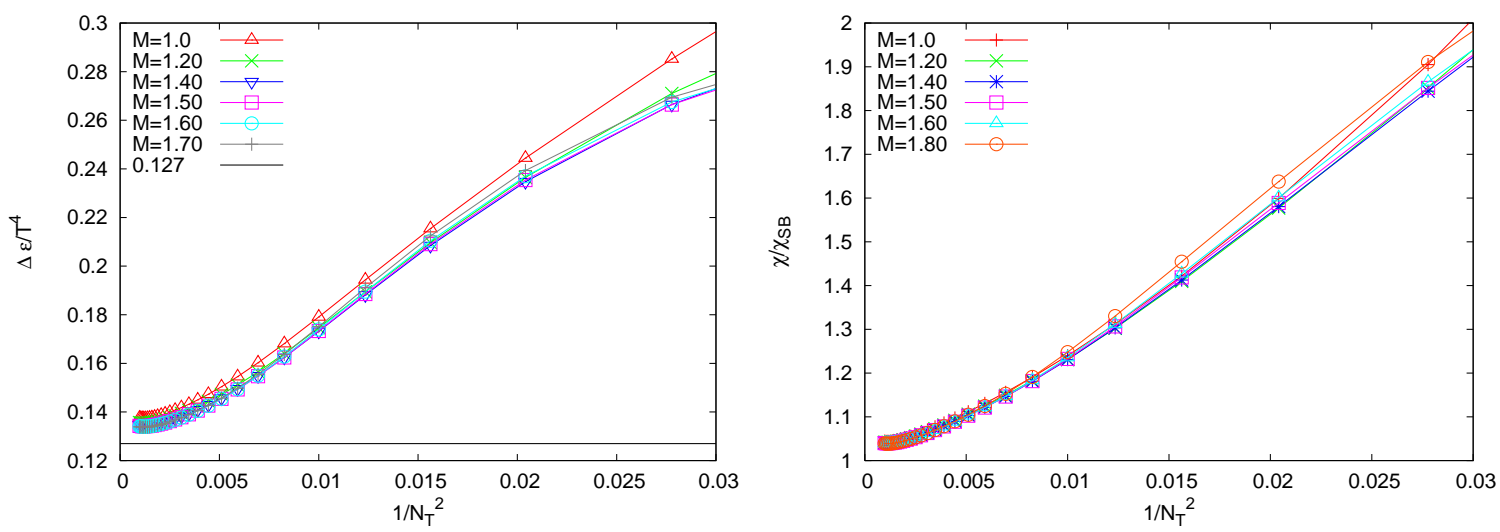

Figure 1: The energy density(left panel) in units of $T^{4}$ and the quark number susceptibility (right panel), normalized to the continuum ideal gas value, as a function of $1 / N_{T}^{2}$ for $M$ values as indicated for lattices with aspect ratio four.

Spurred by the results of the previous sections, we propose two simple ideas for simulations of lattice QCD at finite density. Let us state them at the outset: 1) If chiral invariance and the anomaly relation are to be only restored in the continuum limit, one may introduce the chemical potential on the lattice in a simpler way and 2) known techniques in the literature to eliminate the free theory related divergences may simplify the computations.

From the well-known relation between the domain wall fermions and the overlap fermions $[10,11]$, we know that only fermions confined to the domain walls are physical. Introducing a chemical potential only to count them, one has

$$
D_{o v}(\hat{\mu})_{x y}=\left(D_{o v}\right)_{x y}-\frac{a \hat{\mu}}{2 a_{4} M}\left[\left(1-\gamma_{4}\right) U_{4}(y) \delta_{x, y-\hat{4}}+\left(\gamma_{4}+1\right) U_{4}^{\dagger}(x) \delta_{x, y+\hat{4}}\right] .
$$

The chief advantage of eq.(3.1) is that $D_{o v}$ is defined by the usual sign-function for a Hermitian matrix, making the computations simpler. Of course, it too breaks chiral invariance at the same order as the Bloch-Wettig proposal. One has, however, to expect $a^{-2}$-divergences as $a \rightarrow 0$. Following the same prescription which is used for the pressure computation (which diverges at zero temperature as $\Lambda^{4}$ ), we use physical quantities computed on large $N_{T}$ and the same lattice spacing $a$ for subtraction of these divergences. We tested this by considering two physical quantities for the ideal gas, namely, the difference in the energy density due to nonzero $\mu$, defined by $\Delta \varepsilon(\mu, T)=\varepsilon(\mu, T)-\varepsilon(0, T)$ and the quark number susceptibility, $\chi=T / V \partial^{2} \ln \mathscr{Z} / \partial \mu^{2}$.

Figure 1 shows the results for these quantities, where we used $\mu / T=0.5$ for the $\Delta \varepsilon$ and $\mu=0$ for the $\chi$ to compare with our earlier [4] results using the exponential prescription of Bloch-Wettig. 


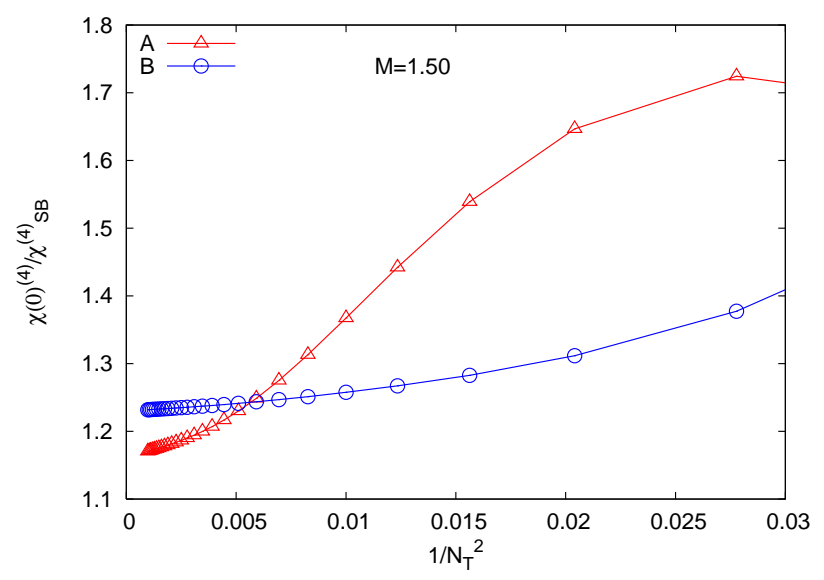

Figure 2: The variation of fourth order susceptibility, normalized by its corresponding continuum value, as a function of $1 / N_{T}^{2}$ for $\zeta=4, M=1.5$ for A) $\hat{\mu} / s$ and B) $\hat{\mu} / M$ way of incorporating the chemical potential.

The zero temperature values were computed numerically on a lattice with a very large temporal extent $N_{T}$ and fixed $a_{4}$ such that $T=1 /\left(N_{T} a_{4}\right) \rightarrow 0$. The Matsubara frequencies then become continuous and hence could be integrated upon numerically. The $M$-values are as indicated along the symbol used. From a comparison of the plots with the corresponding ones [4] for the BlochWettig case, we find that i) the zero temperature subtraction procedure does indeed eliminate the divergences, ii) there are no oscillations for odd-even values of $N_{T}$, iii)the M-dependence is much less pronounced, and iv) the scaling towards the continuum value is linear with the possibility of an easier extrapolation.

A further test of the subtraction procedure is that no additional divergences should be seen in other quantities as well, e.g., the fourth order susceptibility. Figure 2 demonstrates this to be the case. The two curves shown are different possible ways of normalizing the chemical potential term, as indicated. Further improvements in the form to achieve a faster convergence to the continuum needs to be explored.

Taking a cue from eq.(3.1), one can introduce $\mu$ in general by

$$
S_{F}=\sum_{x, y} \bar{\Psi}(x) M(\mu ; x, y) \Psi(y)=\sum_{x, y} \bar{\Psi}(x) D(x, y) \Psi(y)+\mu a \sum_{x, y} N(x, y),
$$

where $D$ can be the the staggered, the Wilson-Dirac or any other suitable fermion operator and $N(x, y)$ is the merely the corresponding point-split and gauge invariant number density. Clearly, as above, one ought to be able to get rid of the annoying divergences by subtracting the same physical quantity computed on large $N_{T}$ and the same lattice spacing $a$. Usually, one would introduce $\exp ( \pm a \mu)$ factors in $M$ and not add the $N$ term. Denoting by superscripted primes various derivatives with respect to $a \mu$, one sees that the usual case has $M^{\prime}=M^{\prime \prime \prime} \ldots=\sum_{x, y} N(x, y)$ and $M^{\prime \prime}=M^{\prime \prime \prime \prime}=M^{\prime \prime \prime \prime \prime \prime \prime} \ldots \neq 0$, i.e, all derivative terms contribute making the successive Taylor coefficients have more and more terms. In contrast, eq.(3.2) leads to $M^{\prime}=\sum_{x, y} N(x, y)$, and $M^{\prime \prime}=M^{\prime \prime \prime}=M^{\prime \prime \prime \prime} \ldots=0$, i.e., a lot fewer terms in the Taylor coefficients. This could potentially help in obtaining higher order coefficients than computed so far. For example, the 4th (8th) order susceptibility, has 4 th (8th) derivative of $M$, which has only one (one) term for eq. (3.2) in contrast 
to five (eighteen!) in the usual case [12]. Whether the subsequent reduction in the computations of $M^{-1}$ actually helps in speeding these computations is currently being investigated by us.

\section{Summary}

We showed, both perturbatively and non-perturbatively, that the introduction of nonzero chemical potential, $\mu$, leaves the anomaly unaffected. The zero modes of the Dirac operator for $\mu=0$ govern it. Nonzero $\mu$ simply scales the eigenvectors, makes the right and left eigenvectors distinct but together they still satisfy a completeness relation. We pointed out further that the reason for this can be traced to a "gauge-like" symmetry in the continuum using which $\mu$-dependent terms can be "gauged" away. All the currently known prescriptions of introducing $\mu$ on lattice can be understood in terms of a similar symmetry on the lattice, and ought to protect the anomaly if the fermion regularization itself has it. While such a symmetry can be shown to exist for local actions, it could be simply adopted for the nonlocal cases such as the overlap fermions. The resultant effective overlap operator for nonzero $\mu$ also satisfies the Ginsparg-Wilson relation but unfortunately is not invariant under the chiral transformation.

We also showed that investigating the overlap fermions at finite density by simply adding the $\mu$-term linearly is feasible. It too has similar chiral symmetry breaking as the Bloch-Wettig proposal but the corresponding inverse propagator is simpler and better-defined. Extending the idea of addition of $\mu$ linearly to the usual staggered fermion case was shown to open a possible avenue for computations of higher order coefficients.

\section{References}

[1] P. Hasenfratz, and F. Karsch, Phys. Lett. B 427, 125 (1983); J. Kogut et al., Nucl. Phys. B 225, 93(1983); N. Bilic and R. V. Gavai, Z. Phys. C23, 77 (1984); R. V. Gavai, Phys. Rev. D32, 519 (1985).

[2] J. E. Mandula, e-Print: arXiv:0712.0651 [hep-lat].

[3] J. Bloch and T. Wettig, Phys. Rev. Lett. 97, 012003 (2006); J. Bloch and T. Wettig, Phys. Rev. D76, 114511 (2007).

[4] D. Banerjee, R. V. Gavai and S. Sharma, Phys. Rev. D78, 014506 (2008).

[5] S. L. Adler, Phys. Rev. 177, 2426 (1969); J. Bell and R. Jackiw, Nuovo. Cim. A51, 47 (1969).

[6] K. Fujikawa, Phys. Rev. 21, 2848 (1980).

[7] M. Kapusta, Finite Temperature Field Theory, 124-125, Cambridge University Press (1985).

[8] R. V. Gavai and S. Sharma, e-Print:arXiv:0906.5188 [hep-lat].

[9] P. H. Ginsparg and K. G. Wilson, Phys. Rev. D25, 2649 (1982).

[10] H. Neuberger, Phys. Rev. D57, 5417 (1998).

[11] R. G. Edwards and U. M. Heller, Phys. Rev. D63, 094505 (2001).

[12] R. V. Gavai and S. Gupta, Phys. Rev. D71, 114014 (2005). 